\title{
Die parallelen Prädikat-Argumentstrukturen der gemeinsamen deutsch-dänischen Wortbildungs- muster und ihre Anwendung im Dänischunterricht
}

\section{The predicate-argument-structures in the common German-Danish word-formation-patterns and their use in Danish-teaching}

\begin{abstract}
In this paper, the author tries to deliver exercises for Polish students of German language with Danish as other foreign language. On the basis of the common German-Danish word-formation-patterns, the author attempts to build a new Danish-learning-model, based on these common patterns, the parallelism of which stays on the level of ca. $60-95 \%$, allowing so a more often German-Danish transfer. Using these and other similarities between the two languages a student of Danish with German as source language can from the very beginning learn Danish in a more effective way.

KEYWORDS: common German-Danish word-formation-patterns, Danish as foreign language, Danish-learning-model for the Germans, German-Danish transfer.
\end{abstract}

\section{GLOTTODIDAKTISCHE EINLEITUNG}

Der Aufsatz versucht, den polnischen Germanistikstudenten mit Deutsch als erster Fremdsprache die Möglichkeit zu eröffnen, während ihres Studiums eine zweite germanische Fremdsprache zu erlernen. Sie können 
dabei u. a. eine der skandinavischen Sprachen wählen. Wenn man auf die Tatsache Bezug nimmt, dass, wenn man die Skandinavischen Sprachen erlernen möchte, die füreinander bekanntlich als keine Fremd-, sondern Nachbarsprachen zu bezeichnen sind, man am besten nur eine auswählen sollte, mit Hilfe von welcher man anschließend zu den sonstigen übergehen kann, so gereicht das Dänische zu der in dieser Hinsicht bestmöglichen Wahl. Einerseits hat man dabei nämlich die schwierigste Aussprache von allen zentralskandinavischen Sprachen als erste erlernt, wonach die übrigen bereits leichter verständlich sind, andererseits bietet die deutsch-dänische Parallelität im Bereich der Wortbildung einem Germanisten einen deutlichen Vorsprung nicht nur gegenüber den Lernern, die des Deutschen unkundig sind, sondern auch gegenüber denjenigen, die eine andere skandinavische Sprache auserwählt haben.

Was die Anfangsphasen des Fremdsprachenunterrichts anbelangt, so ist es durchaus angebracht, den Lerner der L2 nicht nur auf Unterschiede zwischen der Ausgangssprache und der Zielsprache aufmerksam zu machen, sondern auch auf die zwischensprachlichen Gemeinsamkeiten (vgl. Stopyra 2013a: 87ff.). Dies kann ihm dazu verhelfen, sich einen allgemeinen Einblick in die zu beherrschende Sprache zu verschaffen, indem seine Aufmerksamkeit z. B. auf die Lautgestalt der ihm bereits bekannten Internationalismen in der L2 gelenkt wird und in die Art und Weise Einblick gewinnt, wie die L2 mit den Internationalismen grammatisch umgeht, d. h. wie sie von der Schreibung und Morphologie der L2 verarbeitet werden. Der Lerner kann auf die morphologischen und syntaktischen zwischensprachlichen Gemeinsamkeiten hingewiesen werden, ohne die lexikalischen Einheiten der L1 und L2 unter dem Gesichtspunkt zu betrachten, ob sie Entlehnungen darstellen (vgl. weiter unten). Dies kann ihm einen besseren Übergang zur Aneignung der grammatischen und syntaktischen Strukturen der heimischen Lexik der L2 erleichtern. An der Stelle ist noch anzumerken, dass die Bezeichnung L2 im vorliegenden Artikel nicht wörtlich die zweite, sondern de facto die dritte Fremdsprache betrifft, weil er methodische Grundlagen v. a. für die polnischen Germanistikstudenten liefert soll (oder sogar die vierte, wenn man das in den Schulen heutzutage populäre Englische in Betracht zieht), so dass dabei die für die Mehrsprachigkeit charakteristischen Prinzipien zu berücksichtigen sind (vgl. Chłopek 2011).

\section{THEORETISCHE GRUNDLAGE}

Nach dem Modell für syntaktische Kasus und semantische Rollen von Charles Fillmore (1971) werden einzelne semantische Rollen, wie Agens, Pa- 
tiens, Adressat, Rezipient Benefizient usw. typischerweise bestimmten syntaktischen Kasus im Satz zugeordnet, d. h. die agensähnlichste Rolle dem Subjekt, Patiens dem direkten Objekt, der Experiencer, Benefizient und Rezipient dem indirekten Objekt, usw. Die Regel wird gewöhnlich als Linkingregel (vgl. Stopyra 2008a: 107) bezeichnet. Die semantischen Rollen für bestimmte Wortbildungsprodukte lassen sich also am besten nicht separat, sondern in ihrem Satzkontext ermitteln, indem man zu ihrer Identifizierung zugleich auch die Position des betrachteten Wortes im Satz heranzieht, das die gegebene Rolle trägt.

Zugleich werden bestimmte Wortbildungsprodukte von konkreten syntaktischen Größen motiviert, was man am häufigsten nach typischen Motivationsverhältnissen feststellen kann. Die Motivation von Wortbildungsprodukten wird in dem hier gebrauchten Modell somit anhand von der Analyse ihrer Paraphrasen festgestellt. Auch wenn die Paraphrasenmethode vielfach kritisiert werden kann, so stellt sie in der heutigen Sprachwissenschaft nach wie vor ein praktisches und oft gebrauchtes Verfahren dar, mit Hilfe von welchem die Motivation der Wortbildungsprodukte ermittelt werden kann. In dem hier vorgeschlagenen Modell wird jedem untersuchten Wortbildungsprodukt die möglichst einfachste Paraphrase zugeschrieben, welche seine lexikalische Bedeutung und zugleich auch die Bedeutungsbeziehung zwischen seinen unmittelbaren Konstituenten expliziert. Dies ermöglicht auch die Feststellung, von welchem Satzglied (von welchen Satzgliedern) der genannten Paraphrase das untersuchte Wortbildungsprodukt motiviert worden ist. Im vorliegenden Beitrag wird versucht, dies am Beispiel von substantivischen Wortbildungsprodukten zu exemplifizieren, z. B. Fahrer - 'jmd. der fährt', d. h. vom Prädikat motivierter Subjektname (hier: Nomen agentis), Gärtner - 'jmd, der den Garten pflegt', d. h. vom Objekt motivierter Nomen agentis, usw. Das Beschreibungsmodell der Wortbildungsprodukte nach ihrer Prädikat-Argumentstruktur zieht also den syntaktischen Kasus zweimal heran: Einmal als Aushilfe bei der Ermittlung der semantischen Rolle (u. a. je nachdem, als was für ein Satzglied das untersuchte Wort im Satz vorkommt), und ein andermal „intern” bei der Untersuchung der Motivation dieses Wortes durch syntaktische Aktanten seiner Paraphrase.

Die motivierenden Aktanten lassen sich demnach also als bestimmte Satzglieder in der einem bestimmten Wortbildungsprodukt eigenen Paraphrase auffassen, die typischerweise Prädikat und Objekte (Argumente) besitzt, vgl. weiterhin auch z. B. Lehrer - 'jemand, der lehrt' (vom Prädikat motiviertes Nomen agentis); Fischer - 'jmd., der Fische fängt' (vom Objekt motiviertes Nomen agentis); Schreiber - jmd./etw., der/was schreibt' (vom Prädikat motiviertes Nomen agentis/instrumenti). 
Dabei kommen auch noch solche Phänomene zum Vorschein, wie Argumentvererbung und Inkorporation (vgl. Stopyra 2008a: 99ff). Bestimmte deverbale substantivische Suffigierungen und Komposita übernehmen demnach das Argument des Verbs, das sie motiviert, z. B. etw. kaufen > Käufer von etw., etw. tragen > Träger von etw., usw. Das Phänomen ist als Argumentvererbung bekannt. Die vererbten Argumente verlieren in der Regel dabei das Merkmal 'obligatorisch' zugunsten des 'fakultativ'. Sie perkolieren vom motivierenden Ausgangsverb auf das Suffix des substantivischen Zielderivats und damit auf das ganze deverbale Zielderivat. Zugleich werden sie links davon, in demselben Wort, d. h. intern besetzt (Inkorporation), z. B. Hosenträger - 'jmd./etw., der/was Hosen trägt', 'Träger einer Hose' (vom Prädikat motiviertes Nomen agentis/instrumenti mit Objekt als intern besetzter Argumentstelle), Taxifahrer - 'jmd., der Taxi fährt', 'Fahrer eines Taxis' (vom Prädikat motivierter Nomen agentis mit Objekt als intern besetzter Argumentstelle), Allesfresser - 'jmd., der alles frisst' (vom Prädikat motivierter Nomen agentis mit Objekt als intern besetzter Argumentstelle), Romanleser - 'jmd., der Romane liest' (vom Prädikat motivierter Nomen agentis mit Objekt als intern besetzter Argumentstelle), vgl. auch Karten-, Fußball-, Hokkey-, Tennisspieler; Alleskenner, Besenbinder; weiterhin auch Laserdrucker, Dosenöffner, Büstenhalter, Kartenleser (vom Prädikat motiviertes Nomen instrumenti mit Objekt als intern besetzter Argumentstelle, vgl. Stopyra 2008a: 116ff.).

Die Komposita mit Objekt als intern besetzter Argumentstelle werden Rektionskomposita (Eichinger 2000) genannt und ihre Eigenart beruht darauf, dass ihre Interpretation in der Regel nur eine mögliche Option zulässt und somit eindeutig ist (im Unterschied zu Komposita vom Typ Fischfrau, die mehrere (oder gar viele) Lesarten zulassen, vgl. Heringer 1984: 1ff). Die Interpretation der Rektionskomposita als Komposita ist üboigens dann möglich, wenn ihre zweite unmittelbare Konstituente ein im Deutschen/ Dänischen gebräuchliches Wort darstellt (worüber es manchmal jedoch schwer zu urteilen ist). Im negativen Falle, dann also, wenn sich kein gebräuchliches Wort als zweite unmittelbare Konstituente heraussondern lässt, sondern eher ein Suffix, wird von Zusammenbildungen gesprochen, z. B. im Falle von Filmemacher ist eher eine Paraphrase wie 'jmd. der Filme macht' als etwa *'Macher von Filmen' angebracht und somit die Lesart als Zusammenbildung (vgl. Rajnik 1983) wahrscheinlicher, d. h. als Derivat mit dem Suffix -er von der Phrase 'Filme machen' als Basis. Manchmal ist dabei auch jedoch eine doppelte Lesart möglich, z. B. Alkoholfahrer - 'jmd. der unter Einfluss von Alkohol fährt' (Zusammenbildung), oder 'jmd. der (beruflich als Transportunternehmer) Alkohol fährt', d.h. 'Fahrer von Alkohol' (Rektionskompositum). 


\section{DEUTSCH-DÄNISCHE PARALLELEN ALS ANLASS ZUM TRANSFER}

Was die lexikalische Seite eines derart ausgerichteten Unterrichtsmodells anbelangt, so kann auf die zwischen der L1 und L2 bestehenden Parallelen im Bereich der Wortbildung hingewiesen werden. Besonders viele derartige Gemeinsamkeiten bestehen zwischen nahe verwandten Sprachen, z. B. zwischen dem Deutschen und dem Dänischen. Man kann dabei sogar von gemeinsamen deutsch-dänischen Wortbildungsmustern sprechen (vgl. Fredsted u. a. 2005; Stopyra 2006: 688ff.). Die Parallelität, verstanden als die bei bestehender semantischer Äquivalenz zugleich bewahrte formale und distributive Äquivalenz zwischen den Wortbildungsprodukten der L1 und L2, verschafft dem deutschsprachigen Lerner den Eindruck, dass er sich, während er Dänisch spricht, im Bereich der Wortbildungsstrukturen der eigenen Sprache bewegt, die er aus dem Deutschen ins Dänische nur „wörtlich zu übersetzen“ braucht, die also (zumeist) Lehnübersetzungen aus dem Dänischen darstellen. Dieselben Wortbildungsstrukturen wie die deutschen sollten nur mit Hilfe vom dänischen Sprachmaterial ausgefüllt werden. Eine derartige formale Äquivalenz bei zugleich eingehaltener semantischer ist keine seltene Erscheinung, weil sie z. B. auch zwischen den deutschen und polnischen Wortbildungskonstruktionen vorkommt, zwischen dem Deutschen und Dänischen ist sie jedoch häufig erstaunlich hoch und beträgt sogar bis über 90\% (vgl. Stopyra 2008a: 230). Was die dänischen Lehnübersetzungen der deutschen Wortbildungskonstruktionen anbelangt, so kommt außerdem jedoch noch der das Lernen sehr erleichternde Umstand hinzu, dass zwischen ihnen auch substantielle Ähnlichkeit, d. h. Tautonymie herrscht (vgl. Stopyra 2013a: 87ff.). Was die Frage des Übergangs der deutschen Wörter ins Dänische, die Ausgangswörter für Analogiebildungen darstellen, anbelangt, so ist dabei außerdem auch vom Phänomen der direkten Übernahme der deutschen morphologischen Strukturen ins Dänische, ohne ein ins Dänische übernommenes Ausgangswort, zu sprechen (vgl. Stopyra 2008a: 14). Dabei erweist sich die dänische Wortbildung als das sprachliche Subsystem, das zur Übernahme deutscher Wortbildungskonstruktionen am geeignetsten ist (vgl. Stopyra 2011: 292).

\section{METHODISCHE ASPEKTE}

\subsection{Zur Entlehnungsproblematik}

Was die prozentual hohe formale Äquivalenz der deutschen Wortbildungsprodukte und ihrer dänischen semantischen Äquivalente anbelangt, so ist es ein Phänomen, das von der Entlehnungsproblematik durchaus se- 
parat zu behandeln ist. In der vorliegenden Untersuchung ist durchaus von jeweils heimischer deutscher und dänischer Lexik die Rede, d. h. vom Wortschatz, der sich im Gegenwartsdänischen längst eingebürgert hat und der im Bewusstsein der dänischen Muttersprachler somit durchaus als heimisch gilt. Die meisten hier behandelten dänischen Wortbildungsprodukte können aus strukturell-funktionalem Gesichtspunkt also als Lehnübersetzungen der deutschen angesehen werden, wobei aber keine direkten Entlehnungen (im Sinne von Szubert 2003), ebenfalls keine "Internationalismen“, in Betracht gezogen werden.

\subsection{Zu den Begrenzungen in der formalen Äquivalenz}

Die hohe Prozentquote der den deutschen formal äquivalenten dänischen Wortbildungsprodukte bei gleichzeitig eingehaltener semantischer Äquivalenz ist dabei natürlich nicht unbegrenzt. Es gibt Produktivitätsbeschränkungen in der Wirkung einzelner Wortbildungsmuster, z. B. in Bezug auf die Hinzufügung von bestimmten Affixen zu Basen bestimmter Wortart, außerdem lassen sich nicht alle Objekte der den betrachteten Wortbildungsprodukten zugrunde liegenden Phrasen im Deutschen und im Dänischen in gleichem Maße intern besetzen, sondern in einer der Sprachen kann an Stelle einer Zusammenbildung (eines Rektionskompositums) ein ihr semantisch äquivalentes einfaches Derivat vorkommen (vgl. Stopyra 2008a: 234ff). Die Regeln für die Suffixsequenz sind auch streng vorgeschrieben (vgl. Stopyra 2008b: 231ff). Um den Lernern die Tatsachen bewusst zu machen, müsste man ihnen ein Set von derartigen Beschränkungsregeln beibringen. Es gibt aber auch eine andere Methode, die den Lernern dazu verhelfen könnte, sich im Rahmen von gängigen und zulässigen, $d$. h. produktiven Wortbildungsmustern sicherer zu bewegen als im Rahmen der unproduktiven und somit die deutsch-dänische Parallelität nicht zu überschätzen. Die Methode würde auf der Miteinbeziehung von einer möglichst größten Menge von Übungen mit in den Unterrichtsprogramm beruhen, die den zulässigen Bereich trainieren.

Dieses Prinzips für den Aspekt des Unterrichts der dänischen Grammatik scheint sich Rajnik bedient zu haben (vgl. Rajnik 2011). Die in seinem Handbuch zur dänischen Morphologie zusammengestellten Regeln werden jeweils mit einer sehr großen Menge von Beispielen exemplifiziert. Der so mit den im Alltag gebräuchlichen Belegen vertraut gemachte Schüler wird Wortbildungsprodukte, die regelwidrig sind, als fremd empfinden und vermeidet es nachher, sie zu gebrauchen. Sobald er sich das Regelsystem der dänischen Morphologie angeeignet hat, kann er sich sicherlich dann aber 
auch, durch häufigen Gebrauch des Dänischen, besonders im Milieu seiner Muttersprachler, am Produzieren von Augenblicksbildungen nach produktiven Wortbildungsmustern beteiligen, was an seiner Sprachkompetenz bereits eine Stufe höher liegen würde. Erst im Milieu der Muttersprachler nach Erwerbung der höchstmöglichen Sprachkompetenz kann sich der Lerner einer Fremdsprache erlauben, selbstständig Ad-hoc-Bildungen zu erzeugen, in Bezug auf welche er mit großer Wahrscheinlichkeit voraussetzen kann, dass sie in einem konkreten Kontext und Sprechsituation richtig verstanden und akzeptiert werden. Im Anfängerunterricht gilt es jedoch vorerst, wie bereits erwähnt, dem Lerner eine Sprachkompetenz zu vermitteln, mit Hilfe von welcher er sich im Bereich von mehr oder minder produktiven Wortbildungsmustern, d. h. im zulässigen Geltungsbereich der deutsch-dänischen formalen Äquivalenz, sicher bewegen kann.

\section{DIE AUF DER BASIS DER FORMAL ÄQUIVALENTEN DEUTSCH-DÄNISCHEN WORTBILDUNGSPRODUKTE KONSTRUIERTEN ÜBUNGEN}

Die in den bisherigen Kapiteln zusammengestellten Prinzipien erlauben es, nicht nur die deutsch-dänische formale Äquivalenz bei gleichzeitig eingehaltener semantischer, d. h. das Hinzutreten von analog funktional ausgerüsteten Affixen zu bestimmten Basen bei Derivaten, das Vorhandensein entsprechender Sequenz von den unmittelbaren Konstituenten bei den Zusammensetzungen usw., zu bewahren, sondern jeweils auch die semantische Rolle zu berücksichtigen, die einem bestimmten Wortbildungsprodukt zukommt und den syntaktischen Aktanten, der ein bestimmtes Wortbildungsprodukt motiviert (vgl. Stopyra 2008a: 121ff.). Eines der Ziele der vorliegenden Untersuchung beruht somit darauf, glottodidaktische Voraussetzungen zur Beantwortung der Frage zu schaffen, inwieweit die Berücksichtigung der Analyse der Wortbildungsprodukte nach ihrer PrädikatArgumentstruktur die Übungen bereichert, die auf der einfachen Teilung von Wortbildungsprodukten in Morpheme beruhen. Somit gilt es, nicht nur die in Handbüchern zu Wortbildung häufig geübte Morphemstruktur der Wortbildungsprodukte, sondern auch ihre Prädikat-Argumentstruktur in die Praxis der Übungen für den Anfänger- und Frühe-Mittelstufe-Unterricht des Dänischen für die des Deutschen kundigen Lerner umzusetzen. Dabei kann das Dänische, wie bereits erwähnt, sowohl die Zweitsprache der Lerner, als auch ihre Drittsprache (d. h. zweite Fremdsprache) darstellen. Demnächst folgen Vorschläge für konkrete Übungen, die die theoretischen Grundlagen des vorliegenden Aufsatzes berücksichtigen, nach Möglichkeit 
mit bewahrter Gradation ihres Schwierigkeitsgrades, d. h. angefangen von den einfachsten zu den mehr komplizierten. Die sämtlichen Übungen sind in Anlehnung an die Untersuchung von Stopyra (2008a: 121ff) durchgeführt worden. Bei der allgemeinen Einführung in die mit semantischen Rollen verbundene Thematik kann dem Lerner die erste demnächst angeführte Übung helfen (mögliche Lösungen erscheinen fett gedruckt in eckigen Klammern):

1. Bilden Sie analog nach dem angegebenen Muster Wortbildungsprodukte von derselben Ableitungsbasis:

$\begin{array}{llll} & \text { N. actionis/acti } & \text { N. agentis } & \text { N. patientis } \\ \text { jmdn. prüfen - } & \text { Prüfung } & \text { [Prüfer] } & \text { Prüfling } \\ \text { etw. lehren - } & \text { Lehre } & \text { Lehrer } & \text { [Lehrling] } \\ \text { jmdn./etw. senden } & \text { [Sendung] } & \text { Sender } & \text { Sendling, usw. }\end{array}$

2. Bilden Sie analog gebaute dänische Agensbezeichnungen nach angegebenen deutschen:

Beispiel:

'jmd. tanzt' > Tänzer 'ngn. danser' > danser

a) 'jmd. raucht' $>$ Raucher 'ngn. ryger' $>$ [ryger]

b) 'jmd. lehrt etw.' > Lehrer 'ngn. lærer ngt.' > [lærer]

c) 'jmd. klagt jmdn. an' > Ankläger 'ngn. anklager ngn.' > [anklager], usw.

2a)

a) 'jmd. siedelt um' > Umsiedler 'ngn. flytter om' > [omflytter]

b) 'jmd. fängt etw. an' > Anfänger 'ngn. begynder med ngt.' > [begynder]

c) 'jmd. verteidigt jmdn.' > Verteidiger 'ngn. forsvarer ngn.' > [forsvarer], usw.

\section{2b)}

Beispiel:

'jmd. gibt Arbeit' > Arbeitgeber 'ngn. giver arbejde til ngn.' > arbejdsgiver

a) 'jmd. trägt eineFahne' > Fahnenträger ' 'ngn. bærer en fane' > [fanebærer]

b) 'jmd. deckt ein Dach' > Dachdecker 'ngn. tækker taget' $>$ [tagtækker], usw.

3. Bilden Sie analog gebaute dänische Agensbezeichnungen nach angegebenen deutschen:

Beispiel:

'jmd. betreibt Politik' > Politiker 'ngn. bedriver politik' > politiker 
a) 'jmd. betreibt Kunst' > Künstler 'ngn. bedriver kunst' > [kunstner]

b) 'jmd. verlegt Bücher' > Verleger 'ngn. ejer el. driver et forlag' > [forlægger] usw.

4. Bilden Sie analoge dänische Tätigkeitsbezeichnungen nach angegebenen deutschen:

Beispiel:

'jmd. stellt etw. auf' > Aufstellung 'ngn. stiller ngt. op' > opstilling

a) 'jmd. erzählt jmdm. etw.' > Erzählung 'ngn. fortæller ngn. ngt.'> [fortælling]

b) 'jmd. meint etw.' > Meinung 'ngn. mener ngt.' > [mening]

c) 'jmd. erinnert sich an etw.' > Erinnerung 'ngn. erindrer ngt.' > [erindring] usw.

5. Bilden Sie analoge dänische Tätigkeitsbezeichnungen nach angegebenen deutschen:

Beispiel:

'jmd. besetzt etw.' > Besetzung 'ngn. besætter ngt.' > besættelse

a) 'jmd. übersetzt etw.' > Übersetzung 'ngn. oversætter ngn.' >

[oversættelse]

b) jmd. verbindet etw. mit etw. > Verbindung 'ngn. forbinder ngt. med ngt.' >

c) 'jmd. erwägt etw.' > Erwägung 'ngn. overvejer ngt.' > [overvejelse] usw.

6. Bilden Sie dänische Bezeichnungen für Resultate von Tätigkeiten analog nach angegebenen deutschen:

Beispiel:

'jmd. versäumt etw.' > Versäumnis 'ngn. forsømmer ngt.' > forsømmelse

a) 'jmd. gesteht etw.' > Geständnis 'ngn. tilstår ngt.' > [tilståelse]

b) 'jmd. erlebt etw.' > Erlebnis 'ngn. oplever ngt.' > [oplevelse]

c) 'jmd. versäumt etw.' > Versäumnis 'ngn. forsømmer ngt.' > forsømmelse] usw.

7. Bilden Sie dänische Bezeichnungen für Resultate von Tätigkeiten analog nach angegebenen deutschen: 
Beispiel:

'jmd. gibt etw. an' > Angabe

'ngn. angiver ngt.' > angivelse

a) 'jmd. nimmt etw. an' > Annahme 'ngn. antager ngt.' > [antagelse]

b) 'jmd. gibt die Zeit an' > Zeitangabe 'ngn. angiver tiden' > [tidsan-

givelse] usw.

8. Bilden Sie analoge dänische Eigenschaftsbezeichnungen nach angegebenen deutschen:

Beispiel:

'jmd. ist frei' > Freiheit

'ngn. er fri' > frihed

a) 'etw. ist schön' > Schönheit

'ngt. er skønt' > [skønhed]

b) 'jmd. ist dankbar' > Dankbarkeit

c) 'etw. ist rein' > Reinheit

'ngn. er taknemmelig' > [taknemme-

lighed]

'ngt. er rent' > [renhed] usw.

\section{ZUSAMMENFASSUNG DER ÜBUNGSERGEBNISSE}

Es wird davon ausgegangen, dass die oben angeführten Übungen nur einen Vorschlag darstellen und dass durchaus auch andere Optionen ihrer Gestaltung in Frage kommen. Die meisten der eben präsentierten Übungsvorschläge trainieren jeweils nur eine semantische Rolle, welche in der jeweiligen Übung von einem bestimmten syntaktischen Aktanten motiviert ist und mit einem bestimmten Suffix zusammenhängt. In der Übung $3 b$ ) unterscheidet sich die jeweils von Muttersprachlern angegebene deutsche Paraphrase von der dänischen, so dass die jeweiligen Zielderivate nicht von gleichen syntaktischen Aktanten motiviert sind, d. h. das deutsche Derivat vom Prädikat und das dänische vom Objekt der ihnen zugrunde liegenden Paraphrase. Ebenfalls in der Aufgabe 4c) ist eine zwischensprachliche Unstimmigkeit sichtbar gemacht worden, die auf einem wichtigen Valenzunterschied zwischen zwei semantisch äquivalenten (und zugleich auch tautonymen) deutsch-dänischen Verben beruht, welcher mit ihrem präpositionalen (im Deutschen) oder transitiven (im Dänischen) Gebrauch verbunden ist. Dem Lerner soll damit auf mögliche Begrenzungen in der Wirkung der deutsch-dänischen Wortbildungsmuster hingewiesen werden. Prinzipiell in gesondert ausgegrenzten Übungen (2b) sind deutsche und dänische Wortbildungsprodukte dargestellt worden, welche vom dem ihnen zugrunde liegenden Prädikat motiviert sind mit gleichzeitiger internen Besetzung 
des Objekts links von diesem Derivat. Ebenfalls extra ausgegrenzt wurden jeweils die deutschen und dänischen Belege, welche zueinander nicht Tautonym sind (2a).

\section{SCHLUSSFOLGERUNGEN}

Die Ausnutzung der prozentual so hohen strukturell-funktionellen Parallelität zwischen den deutschen und dänischen Wortbildungsmustern, welche in der Anfangsphase des Dänischunterrichts zumeist noch unsichtbar ist, scheint selbstverständlich zu sein. Angesichts der ersten Übungsversuche im Anfängerunterricht des Dänischen für die polnischen Germanistikstudenten kann man bereits in dem Augenblick darauf schließen, dass die zusätzliche Konstruierung der Übungen nach der PrädikatArgumentstruktur der den Wortbildungsprodukten zugrunde liegenden Phrasen eine wesentliche Bereicherung und Vervielfältigung der Übungen darstellt im Vergleich zu denjenigen Übungen, wo man die bloße Morphemstruktur der Wortbildungsprodukte trainiert. Man kann in Bezug darauf nur noch hoffen, den Lerner auf Dauer in die mit der fließenden Kenntnis der parallelen deutsch-dänischen Wortbildungsmuster verbundene Sprachkompetenz einzuführen, die mit gleichzeitigem Bewusstsein der Begrenzungen in ihrer Produktivität einhergeht.

\section{LITERATURVERZEICHNIS}

Chłopek, Z., 2011. Nabywanie języków trzecich oraz kolejnych. Aspekty psycholingwistyczne ( $\mathrm{i}$ inne). Wrocław: Wydawnictwo Uniwersytetu Wrocławskiego.

Eichinger, L.M., 2000. Deutsche Wortbildung. Eine Einführung. Tübingen: Narr.

Fillmore, Ch., 1971. Plädoyer für Kasus. In: Kasustheorie. Abraham W. (Hrsg.) (Schwerpunkte Linguistik und Kommunikationswissenschaft, Band 2), Frankfurt am Main, $1-118$.

Fredsted, E., Carstensen, A., Kühl, K., 2005. Projekt: Divergerende sprogbrug hos unge og bilingvale. In: Widell, P., Kunøe, M. (Hrsg.) 10. Møde om Udforskningen af Dansk Sprog. Århus, 113-122.

Heringer, H.J., 1984. Wortbildung: Sinn aus dem Chaos. Deutsche Sprache 12, 1-13.

Rajnik, E., 1983. Unfeste substantivische Zusammenbildungen im Deutschen und Dänischen. Zeszyty Naukowe Wydziatu Humanistycznego Uniwersytetu Gdańskiego. Studia Scandinavica 6, 71-105.

Rajnik, E., 2011. Wortbildung des Substantivs im Dänischen - explizite und implizite Derivation. Poznań: Wydawnictwo Naukowe UAM.

Stopyra, J., 2006. Gemeinsame Wortbildungsmuster des Deutschen und Dänischen im Bereich substantivischer Ableitungen. In: Kozmová, R. (Hrsg.). Sprache und Sprachen 
im mitteleuropäischen Raum. Vorträge der internationalen Linguistik-Tage Trnava 2005. Trnava: Filozofická fakulta, Univerzita sv. Cyrila a Metoda v Trnave, 687-693.

Stopyra, J., 2008a. Nominale Derivation im Deutschen und im Dänischen (= Acta Universitatis Wratislaviensis No 3051. Germanica Wratislaviensia 128). Wrocław: Wydawnictwo Uniwersytetu Wrocławskiego.

Stopyra, J., 2008b. Zu der Suffixsequenz im Deutschen und im Dänischen. In: Bukowski, P., Pietrzak-Porwisz, G., Kowal I. (Hrsg.). Perspektiv på svenska språket och litteraturen. Kraków: Wydawnictwo Uniwersytetu Jagiellońskiego, 231-235.

Stopyra, J., 2009a. Einteilung der deutschen und dänischen Derivate nach ihrer PrädikatArgumentstruktur. In: Helin I. (Hrsg.). Käännöstieteen laitoksen julkaisuja VI. Veröffentlichungen des Instituts für Translationswissenschaft VI. Linguistik und Übersetzung in Kouvola. Beiträge zu Sprache und Sprachen 7. Helsinki: Institut für Translationswissenschaft, Universität Helsinki, 275-285.

Stopyra, J., 2009b. Program nauczania języka duńskiego dla osób znających język niemiecki. In: V Międzynarodowa Konferencja Edukacyjna "Języki obce w kontekście wspótczesnych wyzwań i perspektyw". Ustroń, 24-26 IV 2009. Katowice: Studium Praktycznej Nauki Języków Obcych Politechniki Śląskiej (płyta CD).

Stopyra, J., 2010a. Unterrichtsprogramm für Dänisch lernende Deutsche. In: Aspekte der Sprachwissenschaft: Linguistik-Tage Jena. 18. Jahrestagung der Gesellschaft für Sprache und Sprachen e. V. (= Schriftenreihe Philologia. Sprachwissenschaftliche Forschungsergebnisse, Band 144). Hamburg: Verlag Dr. Kovač, 345-351.

Stopyra, J., 2010b. Problemy pracy nad słownictwem w wybranych podręcznikach do języka niemieckiego. In: Droździał-Szelest, K. (Hrsg.). Materiały edukacyjne w nauczaniu języków obcych - teoria i praktyka. Gorzów Wielkopolski, 91-95.

Stopyra, J., 2011. Über das Deutsche zum Dänischen: Ein Umweg für polnische Germanistikstudenten mit Dänisch als zweiter Fremdsprache. In: Białek, E., Huszcza, K., Lipiński, C. (Hrsg.). Texte in Bewegung setzen. Aufsätze zur Literatur- und Fremdsprachendidaktik. Wrocław-Dresden: Quaestio/Neisse Verlag, 291-295.

Stopyra, J., 2013a. Tautonymie zu Beginn des Fremdsprachenunterrichts. In: Quo vadis Fremdsprachendidaktik? Zu neuen Perspektiven des Fremdsprachenunterrichts (= Lingua. Fremdsprachenunterricht in Forschung und Praxis, Band 25). Hamburg: Verlag Dr. Kovač, 87-92.

Stopyra, J., 2013b. Vom Objekt motivierte Subjektnamen im Polnischen und im Deutschen. In: Studia Translatorica. Kompetenzen des Translators. Theorie - Praxis - Didaktik. Wrocław-Dresden: ATUT/Neisse Verlag, 211-219.

Szubert, A., 2003. Englische Entlehnungen im Dänischen. Morphologie und Wortbildung. Poznań: ad rem. 